\title{
Developing Leadership in Managers to Facilitate the Implementation of National Guideline Recommendations: A Process Evaluation of Feasibility and Usefulness
}

\author{
Malin Tistad ${ }^{1,2,3^{*}}$, Susanne Palmcrantz ${ }^{2,4}$, Lars Wallin ${ }^{1,2,5}$, Anna Ehrenberg ${ }^{1,6}$, Christina B. Olsson ${ }^{7,8}$, Göran \\ Tomson ${ }^{9}$, Lotta Widén Holmqvist ${ }^{7,10}$, Wendy Gifford ${ }^{11}$, Ann Catrine Eldh ${ }^{1,2}$
}

\begin{abstract}
Background: Previous research supports the claim that managers are vital players in the implementation of clinical practice guidelines (CPGs), yet little is known about interventions aiming to develop managers' leadership in facilitating implementation. In this pilot study, process evaluation was employed to study the feasibility and usefulness of a leadership intervention by exploring the intervention's potential to support managers in the implementation of national guideline recommendations for stroke care in outpatient rehabilitation.

Methods: Eleven senior and frontline managers from five outpatient stroke rehabilitation centers participated in a fourmonth leadership intervention that included workshops, seminars, and teleconferences. The focus was on developing knowledge and skills to enhance the implementation of CPG recommendations, with a particular focus on leadership behaviors. Each dyad of managers was assigned to develop a leadership plan with specific goals and leadership behaviors for implementing three rehabilitation recommendations. Feasibility and usefulness were explored through observations and interviews with the managers and staff members prior to the intervention, and then one month and one year after the intervention.

Results: Managers considered the intervention beneficial, particularly the participation of both senior and frontline managers and the focus on leadership knowledge and skills for implementing CPG recommendations. All the managers developed a leadership plan, but only two units identified goals specific to implementing the three stroke rehabilitation recommendations. Of these, only one identified leadership behaviors that support implementation.

Conclusion: Managers found that the intervention was delivered in a feasible way and appreciated the focus on leadership to facilitate implementation. However, the intervention appeared to have limited impact on managers' behaviors or clinical practice at the units. Future interventions directed towards managers should have a stronger focus on developing leadership skills and behaviors to tailor implementation plans and support implementation of CPG recommendations.

Keywords: Evidence-Based Practice (EBP), Facilitation, Implementation, Leadership, Management, Stroke Rehabilitation

Copyright: ๑ 2016 by Kerman University of Medical Sciences

Citation: Tistad M, Palmcrantz S, Wallin L, et al. Developing leadership in managers to facilitate the implementation of national guideline recommendations: a process evaluation of feasibility and usefulness. Int J Health Policy Manag. 2016;5(8):477-486. doi:10.15171/ijhpm.2016.35
\end{abstract}

Article History:

Received: 6 July 2015

Accepted: 27 March 2016 ePublished: 11 April 2016

*Correspondence to:

Malin Tistad

Email: mti@du.se

\section{Key Messages}

Implications for policy makers

Despite growing evidence that managers have a vital role in implementing evidence-based practice (EBP) in healthcare, there is a need for further knowledge of how managers can facilitate guideline implementation. Our findings suggest that:

- Managers need support in increasing their understanding and awareness of their vital role in facilitating the implementation of guidelines.

- Leadership interventions should encompass managers at different levels of organisations.

- The Gifford leadership model aids developing interventions that focus on managers' behaviors.

- Data on the process is needed to capture what works, for whom, and in what context when evaluating feasibility in implementation.

- Pilot studies preceding larger implementation studies are useful to ensure both intervention and data collection feasibility.

Implications for the public

To this day, changes in healthcare are a major challenge; many of today's errors and adverse events could be prevented by using evidence-based practice (EBP). However, implementing EBP is easier said than done, and a complete picture as to what facilitates this process is yet to come. One aspect considered vital is the behavior of managers to facilitate guideline implementation. In this study, we piloted a leadership intervention based on a particular theoretical model to support managers in developing implementation plans. We found that the managers appreciated the model, and found it relevant to their practice. However, few implementation plans reflected their own role in facilitating the implementation of stroke rehabilitation guidelines. Further studies should provide supplementary support to managers, in order to facilitate their guideline implementation. 


\section{Background}

Leadership has been suggested as critical in the implementation of evidence-based practice (EBP) among health professionals. ${ }^{1-4}$ EBP involves the ethically sound application of research-based knowledge and includes the use of clinical practice guidelines (CPG) in clinical and organizational decision-making. In this study, leadership is conceptualized as "a multidimensional process of influence to enable staff members to use research evidence in clinical practice, and includes behaviors and activities of managers that exert direct or indirect influence on individuals, their environment, and organizational infrastructure" ( $p$ 128). ${ }^{2}$ Distinctions between leadership and management imply that management focuses more on planning, organizing, and controlling whereas leadership focuses on innovation and change. However, distinctions are often blurred and effective managers apply functions of both leadership and management when implementing change. ${ }^{2,5}$ Consistently, leadership activities suggested to support EBP involve managerial support, policy revision, and auditing. ${ }^{2} \mathrm{~A}$ recent theory on middle managers' role in implementation suggests that activities considered important in influencing effectiveness involve how managers diffuse and synthesize information about the innovation, mediate between the implementation strategy and day-to-day activities, and promote the innovation. ${ }^{6}$ Still, there is limited knowledge on how managers facilitate effective implementation and even less is known about the function and impact of interventions in the development of leadership in front line management regarding the promotion and support of the implementation of CPGs.

CPGs provide recommendations for clinical practice, and are established means to inform EBP. ${ }^{7,8}$ However, access to CPGs alone does not ensure $\mathrm{EBP}^{9,10}$ and health professionals' adherence to guidelines varies greatly. ${ }^{8}$ In Sweden, for example, only $6 \%-20 \%$ of the stroke victims received home-based rehabilitation in 2011, ${ }^{11}$ despite home-based rehabilitation being a top priority in the national stroke guidelines. ${ }^{12}$ Adherence to both acute care and post-stroke guidelines has been associated with improved patient outcome, including improved physical function. ${ }^{13}$ Thus, there is a need for effective strategies to facilitate the implementation of CPGs in stroke rehabilitation. ${ }^{14}$

Previous trials directed towards managers, evaluating interventions for influencing staff members' use of CPG recommendations, indicate a complex undertaking; however, to date, the knowledge is scarce, providing for no certain conclusions. ${ }^{2,15}$ Whereas Hodnett et $\mathrm{al}^{16}$ found no difference between intervention and control sites in a study that included managers and opinion leaders to implement EBP change, Gifford et $\mathrm{al}^{15}$ found significant changes in nurses' use of CPG recommendations in an intervention study directed at clinical and management leadership teams. Gifford et $\mathrm{al}^{15}$ applied a leadership model, "the Gifford model," that focuses specifically on leadership behaviors and activities to support the implementation of CPGs. ${ }^{15,17,18}$ The model includes a taxonomy of leadership behaviors categorized as relation-, change-, and task-oriented behaviors. ${ }^{19}$ More specifically, relation-oriented behaviors include concerns for the provision of support, recognition, and encouragement of mutual trust and cooperation among team members. Change-oriented behaviors, on the other hand, focus on envisioning change, understanding the need for change, and encouraging efforts to implement change. Lastly, task-oriented behaviors involve organizing and planning work activities, clarifying roles and responsibilities, and following up on performance. ${ }^{19,20}$ The use of all three types of behaviors contributes to effective leadership ${ }^{2,15,17,19}$ for implementing CPGs. ${ }^{15,18}$ Inspired by the study by Gifford et al and the model proposed, we developed a leadership intervention to support managers to implement national guidelines for stroke care in outpatient rehabilitation. An intervention aiming to support managers in the implementation of CPG recommendations is supposedly complex, due to its potential influence on and interaction with the local context, and the behaviors required from both those who deliver and those who receive the intervention. ${ }^{21}$ Conducting a pilot and/or feasibility study, including a process evaluation, is a principal step in the development of a complex intervention, and should preferably be performed prior to studies in which the effectiveness of a complex intervention is evaluated. ${ }^{21}$ A pilot study designed as a process evaluation can help to understand aspects of the feasibility (such as barriers to participation) and the mechanisms of impact. Furthermore, it can improve the design of the intervention and evaluation, and indicate the influence of the context. ${ }^{22}$ As feasibility of evaluation procedures has been described elsewhere, ${ }^{23}$ the aim of this study, designed as a process evaluation, was to explore the feasibility and usefulness of a leadership intervention to support managers' implementation of CPG recommendations for stroke care in outpatient rehabilitation, considering the influence of the context.

\section{Methods}

Design

An exploratory design was used for this process evaluation pilot study, applying qualitative methods before, during, and after the intervention. A process evaluation framework suggested by Moore et al, ${ }^{22}$ depicted in Table 1, was applied throughout the study.

\section{Settings}

The study was conducted in two regions in Sweden: an urban area with about 225000 inhabitants, and a rural area with nearly 10000 inhabitants. All five units providing outpatient rehabilitation to people who had suffered a stroke in these areas participated: two in the urban area and three in the rural area. As with healthcare services in Sweden in general, the units were publicly funded and the senior managers were responsible for the quality of care and delivery of safe, cost effective healthcare services. Due to regional autonomy, the financial conditions and the organization of stroke care differed:

- In the urban area, outpatient rehabilitation units had separate agreements with the regional authority to provide rehabilitation to stroke victims in the patients' homes. A financial reimbursement system was in place and, as a result, the units were reimbursed for each rehabilitation session. Thus, the allied health professionals were expected to provide a certain number of rehabilitation sessions each month to meet budget 
Table 1. Components of the Process Evaluation Framework and Data Sources

\begin{tabular}{lll}
\hline Key Component & Description (Adopted From Moore 2015) & Data Source \\
\hline Context & $\begin{array}{l}\text { Contextual factors which affect (and may be affected by) } \\
\text { implementation, intervention mechanisms, and outcome }\end{array}$ & $\begin{array}{l}\text { Pre-intervention interviews with managers and staff members } \\
\text { Post-intervention interviews with managers } \\
\text { Observations of interactions between managers and staff } \\
\text { members }\end{array}$ \\
Implementation & How intervention delivery is achieved and what is delivered & $\begin{array}{l}\text { Observations during the intervention } \\
\text { Mechanism of impact }\end{array}$ \\
& $\begin{array}{l}\text { Participants' responses to and interactions with the } \\
\text { intervention }\end{array}$ & $\begin{array}{l}\text { Managers' implementation plans } \\
\text { Post-intervention interviews with managers } \\
\text { Observations during the intervention } \\
12-\text { month follow-up with managers }\end{array}$ \\
\hline
\end{tabular}

targets for their unit.

- In the rural area, all units had annual, fixed budgets and separate agreements with the regional authority regarding rehabilitation services. Significant changes had recently been carried out in the region: the municipal healthcare unit had been assigned to provide rehabilitation in patients' homes, whereas the primary care centre now only provided rehabilitation to patients at the center. At the time of the study, the hospital outreach team had just started to operate in the area and was responsible for follow-ups and home-based rehabilitation for all stroke patients within the catchment area.

Sample

Senior and front line managers from each healthcare unit as well as the Community Chief Nurse responsible for healthcare quality in one of the units participated in the intervention and were thus subjected to the data collection. Both front line and senior managers were included, as they all have managerial roles including the operational responsibility for daily practice and the overall responsibility for resources and quality, respectively, and thus, a potential to influence the implementation of CPG recommendations. The Community Chief Nurse will henceforth be included in the term "front line managers." In addition, one physiotherapist and one occupational therapist at each unit (hereafter referred to as "staff members") were targeted in the data collection. As shown in Table 2 participants had been in their positions for varying lengths of time and both men and women were represented. Prior to the study, approval was granted by the regional ethical review board in Stockholm, Sweden. Informed consent was given individually by all participants.

Description of the Leadership Intervention and its Causal Assumptions

The intervention focused on managers' knowledge and skills in effective leadership for implementing CPG recommendations. It was organised as a four-month

Table 2. Managers' and Staff Members' Characteristics

\begin{tabular}{lccc}
\hline & $n$ & $\begin{array}{c}\text { Years in Position } \\
<5 / \geq 5 />10\end{array}$ & Women/Men \\
\hline Managers & 5 & $2 / 1 / 2$ & $8 / 3$ \\
$\quad$ Senior managers & 6 & $1 / 4 / 1$ & \\
$\quad$ Frontline managers & & & $8 / 4$ \\
Staff members & 5 & $3 / 0 / 2$ & \\
$\quad$ Occupational therapists & 5 & $1 / 0 / 6$ & \\
$\quad$ Physiotherapists & 7 & & \\
\hline
\end{tabular}

program including two workshops (of one day each) and two teleconferences between the workshops (of 90 minutes each, after three and six weeks), delivered to the management teams in the urban $(n=4)$ and the rural $(n=7)$ areas. Workshops were held in venues away from participants' work settings, by external facilitator teams including experts in (1) knowledge implementation, (2) healthcare improvement, and (3) stroke rehabilitation. The content and structure were based on the following assumptions:

- The intervention applied a mix of didactic and interactive workshop sessions ${ }^{24}$ originating from critical social science concepts such as consciousness-raising and selfreflection, ${ }^{25}$ presuming that sharing experiences in teams would enhance the learning opportunities.

- For leaders to recognise and manage the complexity and interplay between the factors influencing the uptake of evidence into practice, they need both understanding and templates for action. Building on the Promoting Action on Research Implementation in Health Services framework, PARIHS, we introduced and reflected on the elements evidence, context, and facilitation as significant components for successful implementation, ${ }^{26-28}$ including leadership as a context subelement.

- Furthermore, for the leadership component, we added the Gifford model. ${ }^{15}$ In addition to leadership behaviors, the model proposes an overall structure for implementation, illustrated in Figure 1. The structure has strong resemblance with, for example, the KnowledgeTo-Action cycle $^{29}$ and other healthcare improvement tools available.

- A core element of the intervention was for the managers to develop a leadership implementation plan, preferably based on the Gifford model for implementing the three specific recommendations from the Swedish national CPGs for stroke care ${ }^{12}$ (Table 3) in their units. While the Gifford model focuses leadership behaviors in the implementation process of CPGs, the managers were particularly encouraged to consider their role and opportunities to adapt their behaviors in order to facilitate CPG implementation. We assumed this would provide for enhanced understanding of the function of the evidence, the context and facilitation in implementation processes, and particularly the function of leadership in facilitating uptake of evidence in their contexts.

The detailed content of the different parts of the interventions is presented in Table 4. The Gifford model was translated into Swedish and suggested as a template for outlining the implementation plans. 


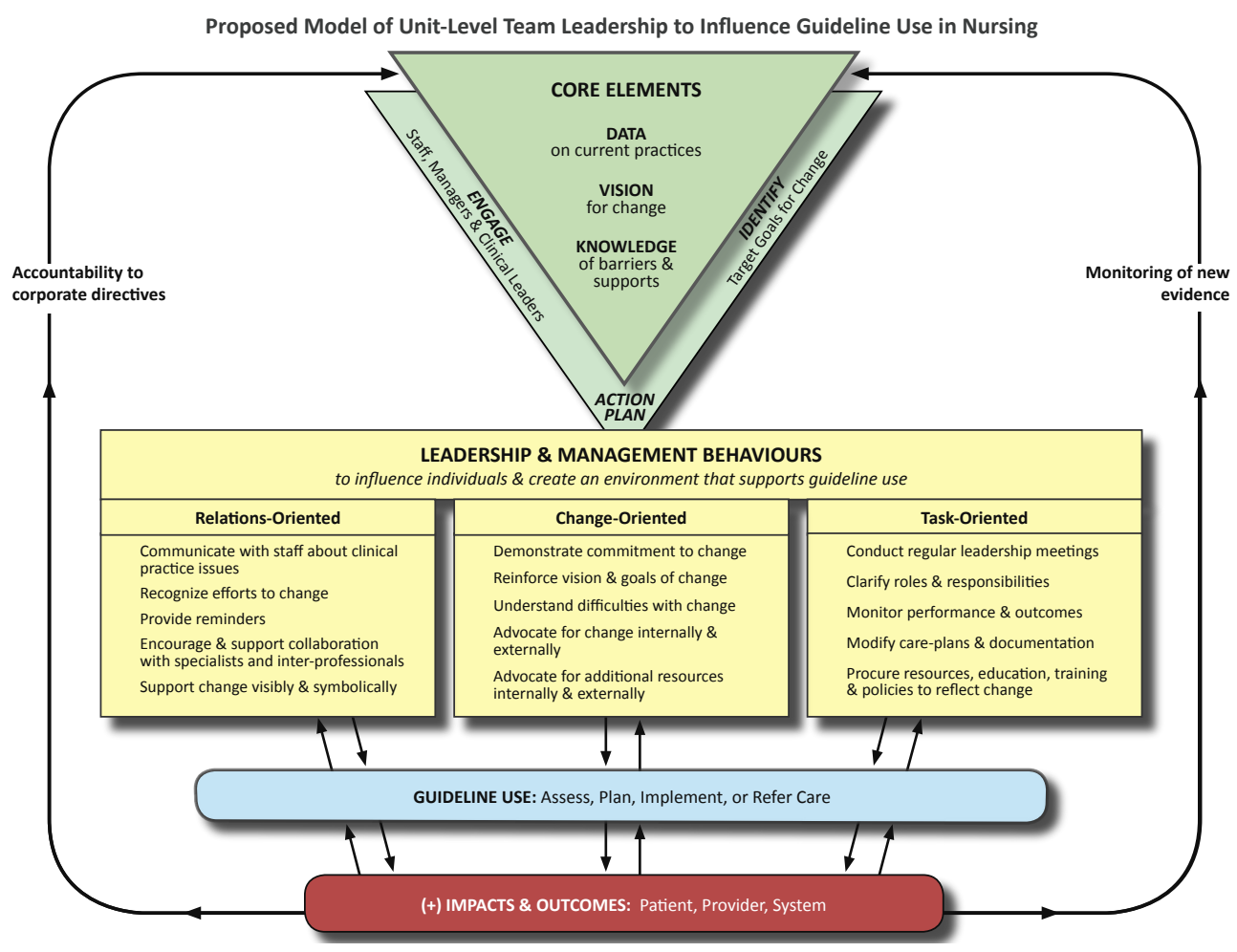

Figure 1. The Gifford Model (Reprinted with permission).

\section{Procedures for Data Collection}

Data were collected in semi-structured interviews and overt non-participant observations with managers and staff members, at time points depicted in Figure 2. For managers, interviews focused on factors known to be important for the process of implementing research evidence ${ }^{30}$ (first interview) and aspects of the intervention including the process of developing a leadership plan for implementation (second interview). Interviews with staff members had additional questions on how daily work was performed (first interview) and staff members' involvement in activities related to the development of the leadership plan (second interview). In addition, a long-term follow-up telephone interview was performed with the frontline managers 9-12 months after the completion of the intervention, focusing on the process of realizing their plans for implementation. While one manager did not want to be audio-recorded but preferred notes to be taken instead, the remaining interviews were audio-recorded and transcribed verbatim.

Observations of interactions between managers and staff members included (1) interactions between front line managers and senior managers; and/or (2) interactions between front line managers and staff members. To ensure that interpersonal interactions were captured, observations were performed during events such as workplace meetings. To capture the intervention process, observations were performed during the workshops and teleconferences. Observations were captured in concurrent field notes. ${ }^{31}$

\section{Data Analysis}

Transcribed interviews and observation texts were analysed using qualitative content analysis. ${ }^{32}$ All transcripts were initially read to obtain "a sense of whole" (p 109), ${ }^{32}$ and thereafter inductively coded by two researchers who had not been engaged in generating or delivering the intervention. The codes were used to identify potential subcategories, which were subsequently abstracted into categories. The categories (with corresponding codes and subcategories) were then deductively analysed considering the key process evaluation components 'context,' 'implementation,' and 'mechanisms of impact. ${ }^{22}$ To complete the analysis, the emerging findings were collated at a conceptual level, illustrating the process evaluation components in relation to outcomes (that is, feasibility and usefulness of the intervention). Trustworthiness was established by recurrent dialogues within the research team regarding the most valid understanding of the data and the rigor of the analysis. ${ }^{33}$

Table 3. The Three Recommendations From the Swedish National Guidelines for Stroke Care ${ }^{13}$

\begin{tabular}{|c|c|}
\hline Recommendation and Priority & Specification \\
\hline $\begin{array}{l}\text { 1) "Training in ADL in the home setting after discharge," } \\
\text { priority } 1\end{array}$ & $\begin{array}{l}\text { Training in ADL in the home setting after discharge, in case of limitations in ADL post stroke. This } \\
\text { limits the risk of unfavorable outcome and improves the ability to perform ADL. }\end{array}$ \\
\hline 3) "Task specific training," priority 3 & $\begin{array}{l}\text { Task specific training aiming to increase activity performance in specified activities among } \\
\text { individuals with impaired movement related function. }\end{array}$ \\
\hline
\end{tabular}

Abbreviation: ADL, activities of daily living. 
Table 4. Content of the Intervention

\begin{tabular}{|c|c|c|c|}
\hline \multirow[b]{2}{*}{ Part of Intervention } & \multirow[b]{2}{*}{ Content } & \multicolumn{2}{|c|}{ Contributing Frameworks } \\
\hline & & $\begin{array}{l}\text { The PARIHS } \\
\text { Framework }\end{array}$ & $\begin{array}{l}\text { The Gifford } \\
\text { Model }\end{array}$ \\
\hline \multirow{4}{*}{$\begin{array}{l}\text { Introduction via } \\
\text { email to each } \\
\text { individual }\end{array}$} & $\begin{array}{l}\text { Two weeks prior to the first workshop, the managers received the three recommendations for } \\
\text { stroke rehabilitation, based on the national guidelines for stroke care. They were further asked to } \\
\text { reflect on: }\end{array}$ & & \\
\hline & - Their experiences of facilitating change; & $x$ & $x$ \\
\hline & $\begin{array}{l}\text { - Their unit and status of staff members' use of the three recommendations and the need for } \\
\text { change; }\end{array}$ & $\mathrm{X}$ & $\mathrm{X}$ \\
\hline & - Potential barriers and facilitators to implementing the three recommendations in their units. & $x$ & \\
\hline \multirow{3}{*}{ Workshop 1} & $\begin{array}{l}\text { Seminars on: } \\
\text { - } \quad \text { Knowledge implementation; } \\
\text { - } \quad \text { Evidence on stroke care; }\end{array}$ & $\mathrm{x}$ & \\
\hline & - $\quad$ Leadership and its potential effect on facilitating the implementation of CPGs into practice. & $\mathrm{X}$ & $\mathrm{X}$ \\
\hline & $\begin{array}{l}\text { The assignment (to develop a leadership plan for the implementation of the three stroke } \\
\text { rehabilitation recommendations) was introduced along with a template of the Gifford leadership } \\
\text { model, suggested as a tool for the assignment. }\end{array}$ & $\mathrm{X}$ & $\mathrm{X}$ \\
\hline Teleconference 1 & $\begin{array}{l}\text { Dialogue, providing opportunities to share experiences of working with the plan/facilitating } \\
\text { implementation of the stroke evidence, and to raise questions and issues regarding the } \\
\text { assignment. }\end{array}$ & $\mathrm{X}$ & $\mathrm{X}$ \\
\hline Teleconference 2 & $\begin{array}{l}\text { Dialogue, providing opportunities to share experiences of working with the plan/facilitating } \\
\text { implementation of the stroke evidence, and to raise questions and issues regarding the } \\
\text { assignment. }\end{array}$ & $\mathrm{X}$ & $\mathrm{x}$ \\
\hline \multirow{3}{*}{ Workshop 2} & $\begin{array}{l}\text { Follow-up on the assignment: the managers shared their leadership plans for implementing the } \\
\text { three recommendations in their units. }\end{array}$ & $\mathrm{x}$ & $\mathrm{x}$ \\
\hline & $\begin{array}{l}\text { A presentation on the model was given via video link by its originator Dr. W. Gifford, including } \\
\text { experiences of projects in Canada and reflection with the participants on their leadership } \\
\text { experiences. }\end{array}$ & & $\mathrm{X}$ \\
\hline & The managers also shared their experiences of the study intervention. & & \\
\hline
\end{tabular}

Abbreviation: PARIHS, Promoting Action on Research Implementation in Health Services; CPG, clinical practice guideline.

Results

Context

Aspects of the context may influence the implementation of the intervention and the mechanisms of impact and hence the feasibility and usefulness of the intervention. Four categories depicted the context of the study units: Provision of rehabilitation; Follow-ups and feedback; Access to evidence and education; and The managers' role.

The provision of rehabilitation was formed by the agreements between the units and the regional authorities. The reimbursement system in the urban area created a time pressure that was perceived to affect the quality and development of rehabilitation negatively, a condition that did not affect the rural units because of their fixed budgets. Moreover, the urban units' staff members and managers depicted limited opportunities to perform tasks beyond what was reimbursed. Rehabilitation itself (ie, the patient-staff interaction) was, to a large extent, shaped by each staff member's knowledge and experience, and staff members' awareness of the national guidelines varied. Meanwhile, managers assumed that staff members' practices were consistent with recommendations of the national guidelines. One unit had an operational management system that involved the systematic development of diagnosis-specific evidence-based protocols and they were about to develop a protocol for rehabilitation after stroke at the time of the study.

Follow-ups and feedback existed to a limited extent; feedback to staff members was primarily focused on the number of rehabilitation sessions carried out. Follow-ups of patient outcomes were performed on an individual level only where the staff member involved 'knew' if a patient had reached his/ her goals for the rehabilitation. Standardized assessment tools were used inconsistently and sporadically.

All units had access to evidence through, for example, the Internet, professional journals, professional networks, students, in-house lectures, and external education. Both managers and staff members considered the recommendations in the national guidelines for stroke relevant and beneficial, but in need of adaptation to the local context before implementation.

Creating beneficial conditions for change was considered part of the managers' role. The frontline managers carried out minor changes in their units but needed senior management's approval for greater modifications. To a large extent, both frontline managers and staff members were autonomous in terms of how they performed tasks. Yet, staff members did not consider managers to be knowledgeable or able to influence the delivery of the rehabilitation.

\section{Implementation}

The component implementation illuminates feasibility and usefulness by describing how the intervention was delivered and what was delivered. The managers' attendance in workshops and teleconferences is displayed in Table 5. Furthermore, the two categories Limited focus on leadership behaviors, and Adaptations of the intervention time plan illustrate the fidelity of the intervention.

Observations during the intervention revealed that there was a limited focus on leadership behaviors among the managers. Discussions about evidence and contextual factors including barriers to implementation were greater priorities for the managers. In one of the teleconferences, managers concluded that they were not yet ready to focus on leadership strategies, a finding confirmed by the lack of outline of leadership 


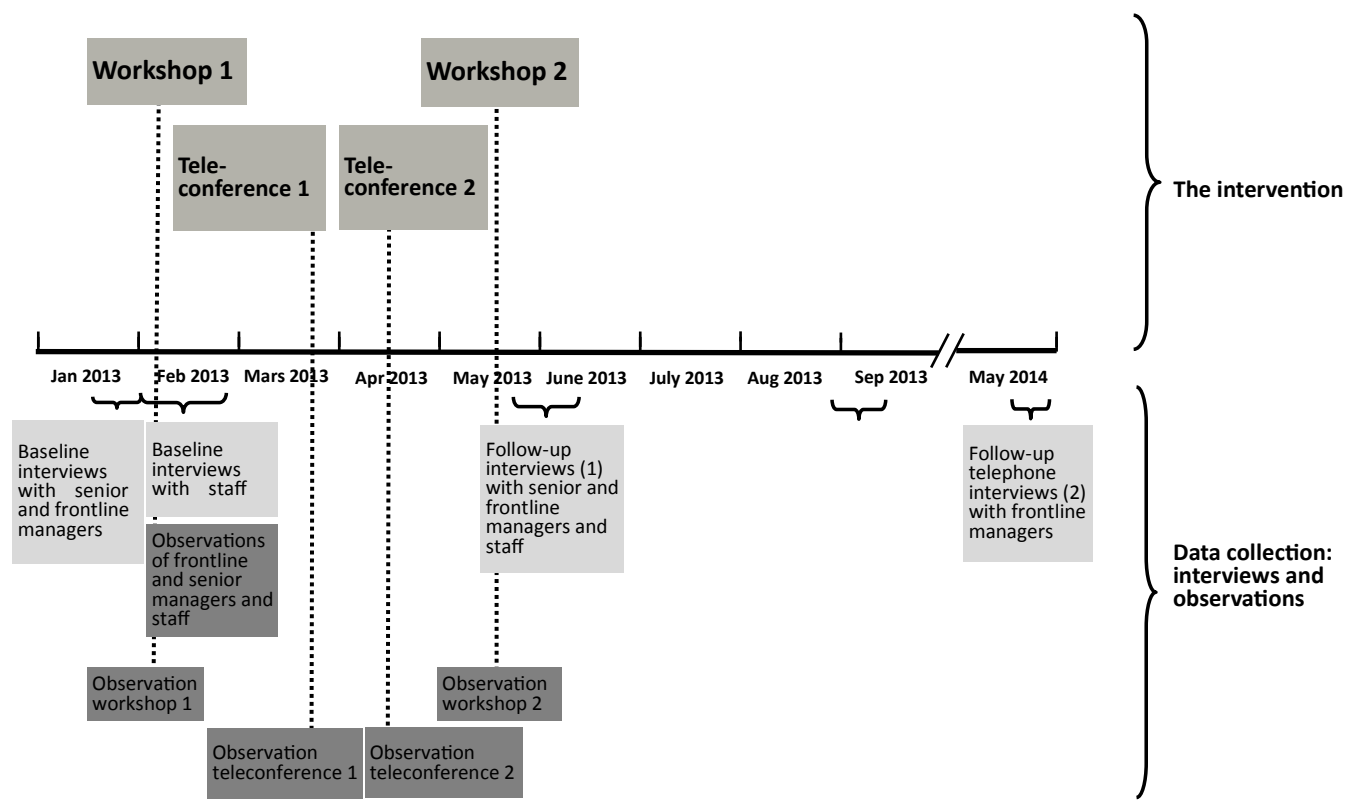

Figure 2. Time Points for the Intervention and Data Collection.

behaviors in their plans. Rather, the managers focused on factors such as: the need for local protocols, collaboration, and time to secure rehabilitation sessions among staff members, as well as for themselves to execute implementation.

Due to external aspects, the delivery of the intervention required Adaptations in terms of the time plan. One teleconference in the urban area was shortened to 60 minutes (rather than 90 minutes), and in the rural area, the second workshop was postponed due to critical management issues in a number of the units. Because of this and summer holidays, the second workshop was delivered six months after the initial workshop (rather than three months, as planned in the program).

\section{Mechanism of Impact: Participants' Response to and Interaction With the Intervention}

Six categories were distinguished that related to how the participants responded to and interacted with the intervention: Content of the intervention; Structure of the intervention; Development of leadership implementation plan; Applicability beyond stroke rehabilitation; Staff involvement; and Leadership for change.

The content of the intervention was perceived to be useful, interesting, and stimulating, and its focus on leadership for facilitating use of CPGs was considered innovative and important. Components that were highly appreciated included the clinical application of theory, for example how national guidelines could be adapted to local clinical conditions, and the translation of the Gifford model into clinical practice. How to operationalize the model, which was described by Gifford during the intervention, was considered to be a core component of the intervention that contributed to participants' understanding of how the model applied to leadership practice.

The structure of the intervention was acceptable to the managers, with an appropriate number of participants and a suitable time frame. The combination of face-to-face workshops and teleconferences worked well but participants thought that the teleconferences should place more focus on how to proceed and develop the leadership implementation plans. The participation of dyads of senior and front line managers from each unit was important: senior managers' attendance provided legitimacy among staff members for the changes that were to be implemented, and was a prerequisite to support frontline managers in their work. Senior managers' participation further meant that they had important insights into the daily work of frontline managers and staff members. All the managers used the Gifford model in the development of their leadership implementation plans, but in different ways:

- The managers of the two urban units identified goals related to the implementation of the target recommendations, ie, standardized assessment tools for stroke patients or development of a local stroke protocol, respectively.

- The three rural units developed a mutual plan that focused on collaboration between them, and identified a general goal phrased as "stroke patients to receive rehabilitation in coherence with the recommendations." In all units, barriers and facilitators were outlined, and a number of activities to address them were included in the plans. However, plans mainly focused on activities to prepare for implementation (for example identifying stroke assessment tools, developing local protocols, or improving collaboration between the units engaged in stroke rehabilitation), rather than actual implementation. Leadership behaviors to support implementation were only outlined in one unit. Frontline managers had the responsibility for developing the plans, while the support of their senior managers varied. In some units, managers interviewed staff members about daily rehabilitation practice and learned that stroke rehabilitation was not carried out as they had anticipated or expected, illustrating an evidence-practice gap.

All managers except one considered the Gifford model had applicability beyond stroke rehabilitation, and that it was a novel structure for supporting implementation.

Although proposed in the intervention, staff involvement in 
Table 5. The Managers' Attendance in Workshops and Teleconferences

\begin{tabular}{|c|c|c|c|c|}
\hline & Workshop 1 & Teleconference 1 & Teleconference 2 & Workshop 2 \\
\hline Frontline managers; attendance in the parts of the intervention (n) & 6 & 6 & 5 & $5.5^{\mathrm{a}}$ \\
\hline Senior managers attendance in the parts of the intervention ( $n$ ) & $3.5^{\mathrm{a}}$ & 3 & 4 & 3 \\
\hline
\end{tabular}

ane participant attended half the day.

developing the implementation plan was limited, with only one out of the 10 staff members interviewed sharing a sense of participation. In one unit, the intervention inspired staff members to reflect on their professional practice, and staff members expressed a desire to discuss this with their managers and colleagues in relation to the stroke recommendations. With regards to leadership for change, front line managers considered having reached their implementation goals as listed in their plan a year after the intervention; one unit had completed the development of a local protocol for rehabilitation after stroke, while managers in another unit had introduced a patient assessment tool, and measures to improve external collaboration had been taken in the three rural units. None of the units made follow-ups to assess the perceived changes, and no further use of the Gifford model was described.

Outcomes, That Is Feasibility and Usefulness, Considering Context and Mechanisms

Illuminating the mechanisms triggered by the intervention, the way it was implemented and the contexts, we propose four key components illustrating the feasibility and usefulness of the leadership intervention:

- The operational management system. The presence of an operational management system that involved a structured process for developing evidence-based protocols for specific diagnosis appeared to be advantageous. The unit using such a system identified leadership behaviors for supporting change in their leadership implementation plan and achieved the implementation goals in the leadership plan.

- $\quad$ The current stroke rehabilitation practice. Implementation of the national guidelines was perceived to have little relevance if: (1) stroke rehabilitation in peoples' homes was not in the unit's assignment, (2) stroke rehabilitation was a small part of the types of healthcare services delivered, or (3) managers considered current rehabilitation practices were already consistent with the recommendations in the national guidelines. Under any of these conditions, managers developed a plan but did not have a clear focus on implementing the recommendations.

- The level of consolidation of the stroke rehabilitation process. In the rural area, the new organization for provision of rehabilitation created an urgent need to establish collaboration between the units, which was reflected in the managers' goals. In the urban area, more stable conditions enabled further development of professional practice by implementing assessment tools and developing a local protocol.

- The timing of the intervention. Most units had other on-going projects or reorganizations taking place at the same time as this project that procured significant time and efforts, thus impacting managers' engagement. Yet, for the unit that was going to develop a clinical protocol for rehabilitation after stroke, timing of the intervention was perceived as highly advantageous and beneficial.

\section{Discussion}

Whereas many studies on implementation of CPGs focus on clinical staff, this study contributes to the understanding of how to support managers in implementation enterprises. The findings of the process evaluation show that although the influence of the leadership intervention on implementation and rehabilitation practice appeared to be limited, the managers appreciated the intervention's intention to support them in the implementation of stroke rehabilitation CPG recommendations. Furthermore, due to its focus on the feasibility of developing managers' leadership behaviors to support implementation, the study suggests that mobilizing knowledge into clinical practices is a complex undertaking. ${ }^{34}$ The frontline managers had a key role and position, situated between senior managers and staff members. These individuals are suggested to be in a central and potentially vital position for facilitating quality improvement and change initiatives, yet they require authority and resources to proceed. ${ }^{6,35,36}$ However, while the senior managers also have a responsibility for the quality of care, they have an important role in facilitating the implementation of CPGs. In this study, the participation of both front line managers and senior managers in the intervention enabled senior managers to support the frontline managers in their implementation efforts. Support from senior managers, including help in prioritizing ${ }^{35,36}$ and the opportunity to discuss problems or successes, ${ }^{37}$ has been reported to be important for front line managers' commitment to implementation. Conversely, a lack of senior managers' support has a direct negative effect. ${ }^{18,37,38}$ Moreover, it is essential that senior managers give continuous encouragement, pay attention to organizational priorities, and make resources available..$^{35-37,39}$ The benefits of including dyads of front line and senior managers in leadership interventions, while explicating their roles within the Gifford model, need further development and emphasis in future studies. Rather, the findings indicate a need for a stronger focus on developing particular leadership behaviors, both in operationalizing the implementation plans, and in supporting managers in applying the behaviors in their management practices. One suggestion is for managers to operationalize the relations, change, and task-oriented leadership behaviors within their work setting as part of the intervention. Significant improvements can be made in leadership knowledge and skills by incorporating formal training interventions into work settings. ${ }^{40}$ Mentoring managers on how to tailor implementation strategies to barriers identified within each unit's specific contexts might also develop specific leadership behaviors for successful implementation. . $^{89}$

Particular context aspects were found to have a negative influence on managers' commitment to the intervention 
assignment, such as if the guideline recommendations corresponded with a unit's mission, and additional major changes were simultaneously taking place. Previous research has shown that the perceived fit between the object of implementation and the units' needs and priorities is of great importance for frontline managers to support implementation, ${ }^{35}$ while additional organizational change impedes implementation, ${ }^{41}$ as does a lack of time. ${ }^{27,36,42}$ Thus, recruitment of units with concurrent involvement in major organizational changes should probably be avoided in implementation studies. ${ }^{41}$ In contrast, a context aspect in the study that seemed to promote the achievement of the goals in the intervention assignment was the presence of a supportive operational management system. In line with this, a previous study has suggested that the presence of a purposeful and active management system supports planning, performing, and follow-up and thus contributes to continuous quality improvements. ${ }^{43}$ Thus, future studies should attend to and explore how the leadership model fits with present management systems, or identify and attend to needs for adjustments.

The insufficient routines for evaluation and feedback to staff members about their clinical performance in some of the participating units could have contributed to the discrepancy in views between managers and staff members regarding guideline recommendations and actual clinical practice. Furthermore, limited knowledge about staff members' adherence to the recommendations and the need for improvement in this may have influenced the managers' engagement in the intervention. ${ }^{35}$ Monitoring staff performance is an essential component of effective leadership, ${ }^{20}$ and can assist with understanding the need for education, training, coaching, and for detecting quality issues. Accordingly, monitoring performances and outcomes has been found to positively influence the use of CPGs. ${ }^{2}$ Consequently, identifying indicators for change that are relevant to each unit's context and developing a strategy to assess these indicators could be part of an intervention to promote leadership engagement. Such performance evaluation could be assigned to managers in collaboration with staff members, to promote evaluation of rehabilitation practices, enable feed-back and enhance staff members' involvement in the implementation process. ${ }^{36,38,39,44}$

Strengths and limitations of the present study should be considered when interpreting the findings. Despite the relatively small number of units included in this pilot study, the sample represents a variety of geographical locations, financial conditions, and ways of organizing outpatient rehabilitation after stroke. Thus, we suggest that the participating units accurately reflect the complexity of today's healthcare system. In the present study, the CPG recommendations were for stroke rehabilitation while the Gifford model was previously tested for the implementation of diabetes CPG recommendations. ${ }^{15}$ Thus, future leadership interventions for implementation using this model should target additional health issues and the guidelines accompanying these issues. Furthermore, while we suggest the process evaluation design and the extensive amount of qualitative data collected provides an understanding of how the intervention was perceived by the managers and the significant contextual influences, quantitative measures on the managers' leadership behaviors alongside the qualitative data would have been a valuable contribution to the understanding of the impact of the intervention.

\section{Conclusion}

In conclusion, we found that the leadership intervention for implementing guideline recommendations, in this case stroke rehabilitation, was delivered in a feasible way (Figure 3). The intervention's focus on leadership for facilitating implementation of CPG recommendations was appreciated by the managers, indicating that this type of intervention has a potential to develop knowledge on change management among healthcare managers. However, the influence of the intervention in this pilot study appeared to be limited on guideline implementation and staff members' rehabilitation practice. Thus, we suggest that a future full-scale trial should incorporate an intervention with a stronger focus on supporting managers to tailor implementation plans to the present context and to apply relevant leadership behaviors to address barriers and support implementation. Evaluation and feedback of staff members' performance and the rehabilitation provided should be considered, and indicators for change relevant to each unit's contextual conditions identified.

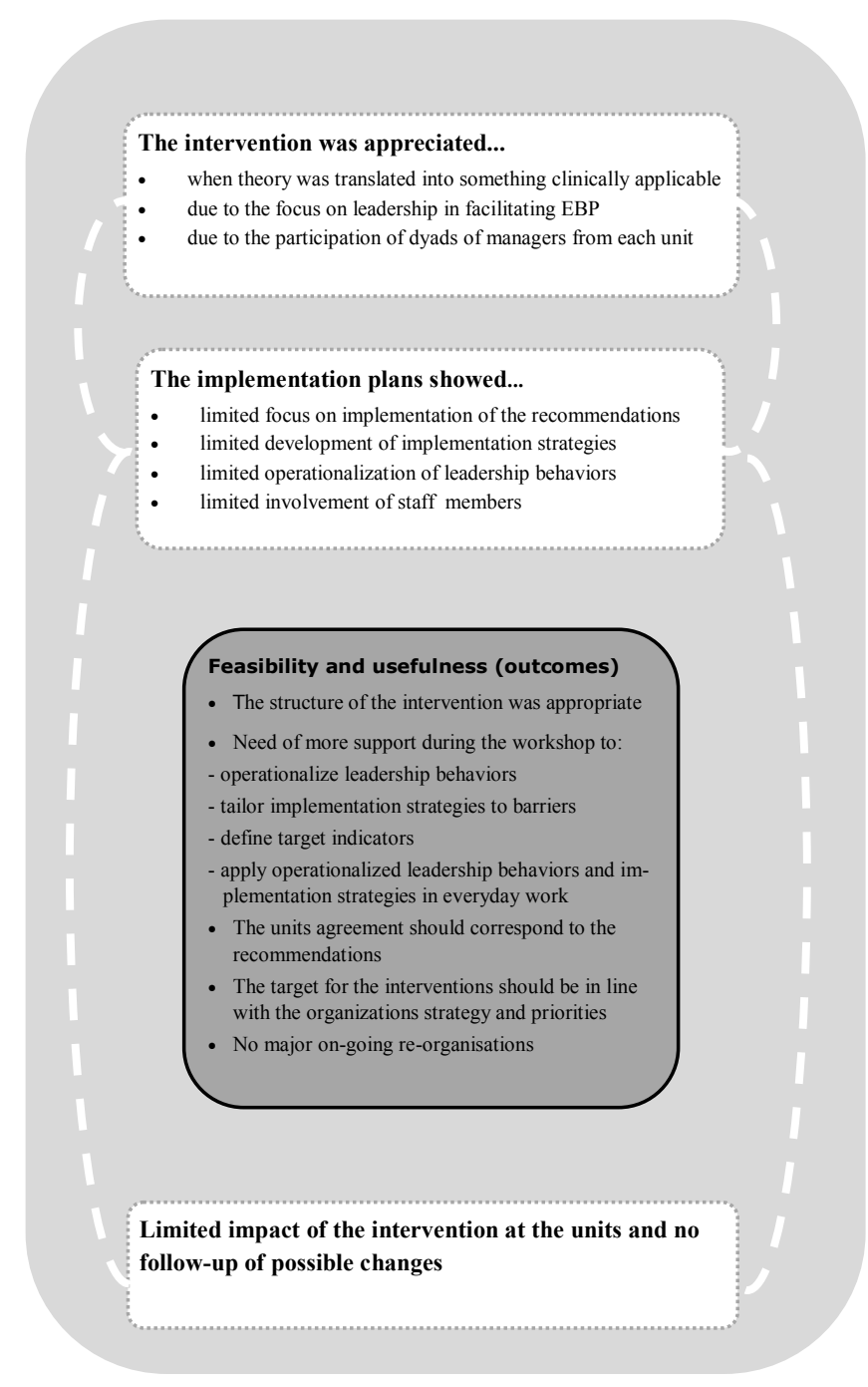

Figure 3. Summary of Conclusions. 


\section{Acknowledgements}

Financial support was provided by the Vardal Foundation, Borås, Sweden and the Swedish Stroke Association, Skärholmen, Sweden (Stroke-Riksförbundet). We are thankful to Disa Sommerfeld who contributed with her extensive knowledge on stroke rehabilitation during the intervention.

Ethical issues

The study was approved by the regional ethical review board in Stockholm, Sweden.

\section{Competing interests}

Authors declare that they have no competing interests.

\section{Authors' contributions}

ACE, LW, GT, AE, and LWH conceived and designed the study. MT and SP acquired the data. MT, SP, and ACE analyzed and interpreted the data. MT, $A E$, and $L W$ drafted the manuscript. ACE, LW, GT, AE, LWH, CO, WG, MT, and $\mathrm{SP}$ contributed with critical revisions of the manuscript for important intellectual content.

\section{Authors' affiliations}

${ }^{1}$ School of Education, Health and Social Studies, Dalarna University, Falun, Sweden. ${ }^{2}$ Division of Nursing, Department of Neurobiology, Care Sciences and Society, Karolinska Institutet, Huddinge, Sweden. ${ }^{3}$ Department of Community Medicine and Rehabilitation, Physiotherapy, Umeå University, Umeå, Sweden. ${ }^{4}$ Department of Clinical Sciences, Danderyd Hospital, Karolinska Institutet, Stockholm, Sweden. ${ }^{5}$ Department of Health and Care Sciences, The Sahlgrenska Academy, University of Gothenburg, Gothenburg, Sweden. ${ }^{6}$ School of Health Sciences, Faculty of Medicine and Health, Örebro University, Örebro, Sweden. ${ }^{7}$ Division of Physiotherapy, Department of Neurobiology, Care Sciences and Society, Karolinska Institutet, Huddinge, Sweden. ${ }^{8}$ Mörby Academic Primary Healthcare Center, Stockholm County Council, Stockholm, Sweden. International Health Systems Research, Departments of Learning, Informatics, Management, Ethics and Public Health Sciences, Karolinska Institutet, Solna, Sweden. ${ }^{10}$ Department of Physiotherapy, Karolinska University Hospital, Stockholm, Sweden. ${ }^{11}$ Faculty of Health Sciences, School of Nursing, University of Ottawa, Ottawa, ON, Canada.

\section{References}

1. Cummings GG, Estabrooks CA, Midodzi WK, Wallin L, Hayduk L. Influence of organizational characteristics and context on research utilization. Nurs Res. 2007;56:S24-S39. doi:10.1097/01.NNR.0000280629.63654.95

2. Gifford W, Davies B, Edwards N, Griffin P, Lybanon V. Managerial Leadership for Nurses' Use of Research Evidence: An Integrative Review of the Literature. Worldviews on Evidence-Based Nursing. 2007;4:126-145. doi:10.1111/j.17416787.2007.00095.x

3. Dannapfel P, Peolsson A, Nilsen P. What supports physiotherapists' use of research in clinical practice? A qualitative study in Sweden. Implement Sci. 2013;8:31. doi:10.1186/17485908-8-31

4. Kristensen HK, Borg T, Hounsgaard L. Aspects affecting occupational therapists' reasoning when implementing researchbased evidence in stroke rehabilitation. Scand J Occup Ther. 2012;19:118-131. doi:10.3109/11038128.2011.556197

5. Jennings BM, Scalzi CC, Rodgers JD 3rd, Keane A. Differentiating nursing leadership and management competencies. Nurs Outlook. 2007;55:169-175.

6. Birken SA, Lee SY, Weiner BJ. Uncovering middle managers' role in healthcare innovation implementation. Implement Sci. 2012;7:28. doi:10.1186/1748-5908-7-28

7. Davies BL. Sources and models for moving research evidence into clinical practice. J Obstet Gynecol Neonatal Nurs. 2002;31:558-562.

8. Grimshaw JM, Eccles MP, Lavis JN, Hill SJ, Squires JE.
Knowledge translation of research findings. Implement Sci. 2012;7:50. doi:10.1186/1748-5908-7-50

9. Grimshaw J, Eccles M, Tetroe J. Implementing clinical guidelines: current evidence and future implications. J Contin Educ Health Prof. 2004;24 Suppl 1:S31-S37. doi:10.1002/chp.1340240506

10. Prior M, Guerin M, Grimmer-Somers K. The effectiveness of clinical guideline implementation strategies--a synthesis of systematic review findings. J Eval Clin Pract. 2008;14:888-897. doi:10.1111/j.1365-2753.2008.01014.x

11. Socialstyrelsen. Nationell utvärdering 2011 - Strokevård Delrapport: Landstingens insatser. (National Assessment 2011 - Stroke Care - County Council Assessment). http://www.socialstyrelsen.se/Lists/Artikelkatalog/ Attachments/18350/2011-6-3.pdf. Accessed October 11, 2015. Published 2011.

12. Socialstyrelsen. Nationella riktlinjer för Strokesjukvård (National Guidelines for Stroke Care). http://www.socialstyrelsen.se/Lists/ Artikelkatalog/Attachments/17790/2009-11-4.pdf. Accessed October 11, 2015. Published 2009.

13. Donnellan C, Sweetman S, Shelley E. Implementing clinical guidelines in stroke: a qualitative study of perceived facilitators and barriers. Health Policy. 2013;111:234-244. doi:10.1016/j. healthpol.2013.04.002

14. Walker MF, Fisher RJ, Korner-Bitensky N, McCluskey A, Carey LM. From what we know to what we do: translating stroke rehabilitation research into practice. Int J Stroke. 2013;8:11-17. doi:10.1111/j.1747-4949.2012.00974.x

15. Gifford WA, Davies BL, Graham ID, Tourangeau A, Woodend AK, Lefebre N. Developing leadership capacity for guideline use: a pilot cluster randomized control trial. Worldviews Evid Based Nurs. 2013;10:51-65. doi:10.1111/j.1741-6787.2012.00254.x

16. Hodnett ED, Kaufman K, O'Brien-Pallas L, Chipman M, Watson-MacDonell J, Hunsburger W. A strategy to promote research-based nursing care: effects on childbirth outcomes. Res Nurs Health. 1996;19:13-20. doi:10.1002/(SICI)1098240X(199602)19:1<13::AID-NUR2>3.0.CO;2-O

17. Gifford W, Davies B, Tourangeau A, Lefebre N. Developing team leadership to facilitate guideline utilization: planning and evaluating a 3-month intervention strategy. J Nurs Manag. 2011;19:121-132. doi:10.1111/j.1365-2834.2010.01140.x

18. Gifford WA, Davies B, Edwards N, Graham ID. Leadership strategies to influence the use of clinical practice guidelines. Nurs Leadersh (Tor Ont). 2006;19:72-88.

19. Yukl G, Gordon A, Taber T. A hierarchical taxonomy of leadership behavior: integrating a half century of behavior research. Journal of Leadership \& Organizational Studies. 2002;9:15.

20. Yukl G. Leadership in Organizations. 8th ed. Boston: Pearson; 2013.

21. Craig P, Dieppe P, Macintyre S, et al. Developing and evaluating complex interventions: the new Medical Research Council guidance. BMJ. 2008;337:a1655. doi:10.1136/bmj.a1655

22. Moore GF, Audrey S, Barker M, et al. Process evaluation of complex interventions: Medical Research Council guidance. BMJ. 2015;350:h1258.

23. Palmcrantz S, Tistad M, Eldh AC, et al. Assessing feasibility and acceptability of study procedures: getting ready for implementation of national stroke guidelines in out-patient health care. BMC Health Serv Res. 2015;15:517.

24. Forsetlund L, Bjorndal A, Rashidian A, et al. Continuing education meetings and workshops: effects on professional practice and health care outcomes. Cochrane Database Syst Rev. 2009;15:CD003030. doi:10.1002/14651858.CD003030. pub2

25. Manley K, McCormack B. Practice development: purpose, methodology, facilitation and evaluation. Nurs Crit Care. 2003;8:22-9. 
26. Kitson AL, Rycroft-Malone J, Harvey G, McCormack B, Seers K, Titchen A. Evaluating the successful implementation of evidence into practice using the PARiHS framework: theoretical and practical challenges. Implement Sci. 2008;3:1. doi:10.1186/17485908-3-1

27. Rycroft-Malone J, Harvey G, Seers K, Kitson A, McCormack $B$, Titchen A. An exploration of the factors that influence the implementation of evidence into practice. J Clin Nurs. 2004;13:913-924. doi:10.1111/j.1365-2702.2004.01007.x

28. Rycroft-Malone J, Kitson A, Harvey G, et al. Ingredients for change: revisiting a conceptual framework. Quality \& Safety in Health Care. 2002;11:174-180. doi:10.1136/qhc.11.2.174

29. Graham ID, Logan J, Harrison MB, et al. Lost in knowledge translation: time for a map? J Contin Educ Health Prof. 2006;26:13-24. doi:10.1002/chp.47

30. Seers K, Linck P, McCarthy G, McCormack B, et al. FIRE (Facilitating Implementation of Research Evidence): a study protocol. Implement Sci. 2012;7:25. doi:10.1186/1748-5908-725

31. Spradley J P. Participant Observation. New York: Holt, Rinehart and Winston; 1980.

32. Elo $\mathrm{S}, \mathrm{Kyngas} \mathrm{H}$. The qualitative content analysis process. J Adv Nurs. 2008;62:107-115. doi:10.1111/j.1365-2648.2007.04569.x

33. Graneheim UH, Lundman B. Qualitative content analysis in nursing research: concepts, procedures and measures to achieve trustworthiness. Nurse Educ Today. 2004;24:105-112. doi:10.1016/j.nedt.2003.10.001

34. Michie S, Johnston M, Abraham C, Lawton R, Parker D, Walker A. Making psychological theory useful for implementing evidence based practice: a consensus approach. Qual Saf Health Care. 2005;14:26-33. doi:10.1136/qshc.2004.011155

35. Chuang E, Jason K, Morgan JC. Implementing complex innovations: factors influencing middle manager support. Health Care Manage Rev. 2011;36:369-379. doi:10.1097/ HMR.0b013e3182100cc2

36. Kirchner JE, Parker LE, Bonner LM, Fickel JJ, Yano EM, Ritchie
MJ. Roles of managers, frontline staff and local champions, in implementing quality improvement: stakeholders' perspectives. J Eval Clin Pract. 2012;18:63-69. doi:10.1111/j.13652753.2010.01518.x

37. Birken SA, Lee SY, Weiner BJ, Chin MH, Chiu M, Schaefer CT. From strategy to action: how top managers' support increases middle managers' commitment to innovation implementation in health care organizations. Health Care Manage Rev. 2015;40:159-168. doi:10.1097/HMR.0000000000000018

38. Ovretveit J. Leading improvement. J Health Organ Manag. 2005;19:413-430. doi:10.1108/14777260510629661

39. Morrow E, Robert G, Maben J. Exploring the nature and impact of leadership on the local implementation of the Productive Ward Releasing Time to Care. J Health Organ Manag. 2014;28:154176. doi:10.1108/JHOM-01-2013-0001

40. Collins DB, Holton EF. The effectiveness of managerial leadership development programs: A meta-analysis of studies from 1982 to 2001. Human Resource Development Quarterly. 2004;15:217-48. doi:10.1002/hrdq.1099

41. Carlfjord S, Lindberg M, Bendtsen P, Nilsen P, Andersson A. Key factors influencing adoption of an innovation in primary health care: a qualitative study based on implementation theory. BMC Fam Pract. 2010;11:60. doi:10.1186/1471-2296-11-60

42. Ploeg J, Davies B, Edwards N, Gifford W, Miller PE. Factors influencing best-practice guideline implementation: lessons learned from administrators, nursing staff, and project leaders. Worldviews Evid Based Nurs. 2007;4:210-219. doi:10.1111/ j.1741-6787.2007.00106.x

43. Eldh AC, Fredriksson M, Halford C, et al. Facilitators and barriers to applying a national quality registry for quality improvement in stroke care. BMC Health Serv Res. 2014;14:354. doi:10.1186/1472-6963-14-354

44. Kristensen HK, Hounsgaard L. Implementation of coherent, evidence-based pathways in Danish rehabilitation practice. Disabil Rehabil. 2013;35:2021-2028. doi:10.3109/09638288.20 13.768301 